\title{
First Results from the Soft X-ray Pulse Height Analysis System on Wendelstein 7-X Stellarator
}

\author{
Monika Kubkowska* ${ }^{a *}$, Agata Czarnecka ${ }^{\mathrm{a}}$, Tomasz Fornal ${ }^{\mathrm{a}}$, Marta Gruca ${ }^{\mathrm{a}}$, Natalia Krawczyk ${ }^{\mathrm{a}}$, \\ Sławomir Jabłoński ${ }^{\mathrm{a}}$, Leszek Ryć ${ }^{\mathrm{a}}$, Henning Thomsen ${ }^{\mathrm{b}}$, Kieran J. McCarthy ${ }^{\mathrm{c}}$, Christoph Biedermann ${ }^{\mathrm{b}}$, \\ Birger Buttenschön $^{\mathrm{b}}$, Arturo Alonso ${ }^{\mathrm{c}}$, Rainer Burhenn ${ }^{\mathrm{b}}$ and the W7-X team \\ ${ }^{a}$ Institute of Plasma Physics and Laser Microfusion, Hery 23, 01-497 Warsaw, Poland \\ ${ }^{b}$ Max Planck Institute for Plasma Physics, 17491 Greifswald, Germany \\ ${ }^{c}$ Laboratorio Nacional de Fusion, CIEMAT, Avenida Complutense, Madrid, Spain
}

\begin{abstract}
The paper presents experimental results obtained during the first operational phase (called OP1.1) of the Wendelstein 7-X stellarator (W7-X). One of W7-X diagnostics that delivers information about plasma impurities and was commissioned during the OP1.1, is the Pulse Height Analysis (PHA) system which provides soft X-ray spectra of highly ionized impurity species over a very broad energy range with energy resolution not worse than $200 \mathrm{eV}$ and with temporal resolution of $100 \mathrm{~ms}$. The line-of-sight integrated spectra, collected by a Silicon Drift Detector in the energy range between 0.9 and $10 \mathrm{keV}$, have been analyzed for various experimental conditions and are discussed with respect to results from other diagnostics. In this work, injected impurities (argon and neon) as well as intrinsic impurities (sulphur and chlorine) have been identified. Spectral lines corresponding to heavier impurities have not been observed.
\end{abstract}

Keywords: PHA system, plasma impurities, Stellarator, soft X-ray plasma diagnostic.

\section{Introduction}

In magnetically confined fusion plasmas, in particular in stellarators, knowledge about impurity content and transport in the plasma is essential information. The Wendelstein 7-X stellarator [2], which was commissioned at the end of 2015, had its first operation phase (OP1.1) at the beginning of 2016 [2-3]. The W7-X belongs to the HELIAS line (HELIcal axis Advanced Stellarator) of devices. It is equipped with a superconducting coil system, designed to operate with a magnetic field of 2.5 $\mathrm{T}$, that is capable of providing a continuous magnetic field, this being a key component for future steady-state operation of the machine. The main objective of the W7$\mathrm{X}$ programme is to prove steady-state plasma operation in a stellarator and demonstrate this option as a candidate for a fusion power plant. For this reason, the study of impurity transport is a key task as avoiding impurity accumulation is required to obtaining long, stable plasma discharges. During OP1.1, 5 poloidal uncooled graphite limiters, located at the inboard side, defined the Last Close Flux Surface (LCFS) of the plasma [2].

During the OP1.1 experimental campaign more than 20 diagnostics were operated and delivered many interesting results [4-5]. One of these was a soft X-ray Pulse Height Analysis (PHA) system [6-7] dedicated mainly to obtaining information about the impurity content in the plasma and an estimation of core electron temperature $\left(T_{\mathrm{e}}\right)$. In many fusion devices, such as tokamaks or stellarators, PHA diagnostics are routinely used during the experiments [8-11]. Indeed, from PHA spectra it is not only possible to provide $T_{e}$ and identify impurities, but also to estimate average effective charge, $Z_{\text {eff }}$ from comparison of experimental and theoretical spectra [9-10].
During OP1.1 only electron-cyclotron-resonance heating (ECRH) was applied (up to 4MW). Initially, discharges with helium were performed, mainly to clean the vacuum vessel. Later, discharges with hydrogen as the working gas were performed. The main plasma parameters achieved were as follows: $\mathrm{T}_{\mathrm{e}}<8 \mathrm{keV}, \mathrm{T}_{\mathrm{i}}<2$ $\mathrm{keV}$ and $\mathrm{n}_{\mathrm{e}}<3 \times 10^{19} \mathrm{~m}^{-3}$ where $T_{\mathrm{i}}$ is ion temperature and $\mathrm{n}_{\mathrm{e}}$ is electron density. Discharges lasting up to $6 \mathrm{~s}$ were achieved [12-13]. It is worth adding that the obtained values were significantly higher than expected [1-2].

\section{Operation of the Pulse Height Analysis System during OP1.1}

A Pulse Height Analysis system equipped with three Silicon Drift Detectors (SDDs) [6-7] has been installed on $\mathrm{W} 7-\mathrm{X}$ to measure continuum and line radiation in the soft X-ray range. A scheme of the diagnostic is shown in Fig. 1. It was commissioned and tested during OP1.1. The optimization of the system required a compromise between energy resolution and count rate performance.

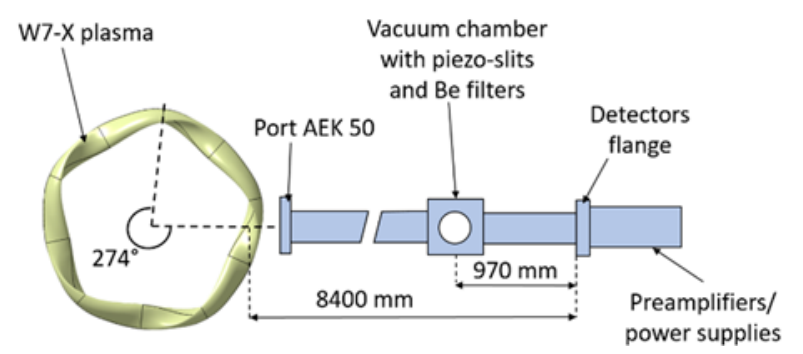

Fig. 1. Schematic showing the principal components of the PHA system at W7-X (not to scale).

As a result of this only the $1^{\text {st }}$ PHA channel was optimized to deliver well-resolved spectra. The analysis and discussion of the spectra presented in this paper is limited to this channel. 
During OP1.1, the $1^{\text {st }}$ PHA channel was equipped with a SDD detector from PNDetector (type SDD-10-130) covered with an $8 \mu \mathrm{m}$ Beryllium (Be) window [14].

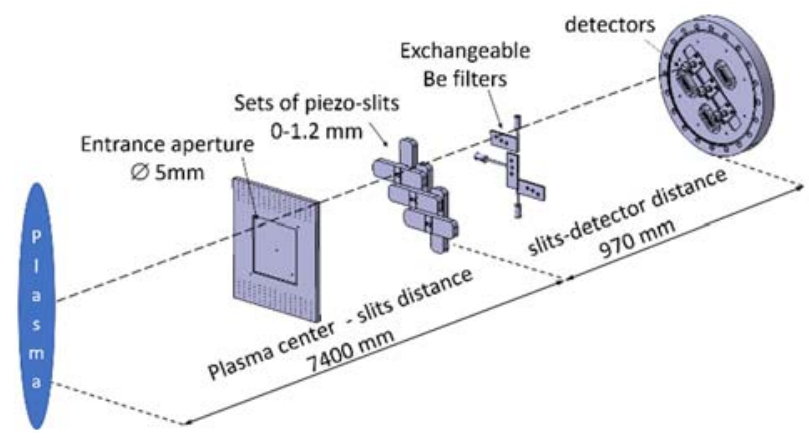

Fig. 2. A scheme showing the arrangement of piezo-slits and additional Be filters in PHA system at W7-X. (not in scale). The entrance aperture which has a function of collimator is located at the entrance of vacuum chamber with piezo-slits and Be filters.

The detector chip dimension is $10 \mathrm{~mm}^{2}$ with a $450 \mu \mathrm{m}$ active layer thickness. The nominal energy resolution, as provided by the producer, is $132 \mathrm{eV} @ 5.9 \mathrm{keV}(\mathrm{Mn} \mathrm{K} \alpha)$ at an operating temperature of $253 \mathrm{~K}$ (Peltier cooler is integrated inside the detector). Additionally, a $25 \mu \mathrm{m}$ thick Be foil was placed in the detector line-of-sight, in order to reduce the photon flux. These defined the energy range of collected spectra from $0.9 \mathrm{keV}$ to $19 \mathrm{keV}$, assuming $37 \%$ (1/exp) detector response. The arrangement of the PHA components is presented in Fig. 2. Pinholes, which define the plasma cone of view, are located about $1 \mathrm{~m}$ from the detector. Next, in order to avoid spectrum distortion a reduction of photon count rate is necessary. For this, a set of remotely control piezo-slits is used. For the results presented here, the slit had a square shape with dimensions $0.3 \times 0.3 \mathrm{~mm}^{2}$.

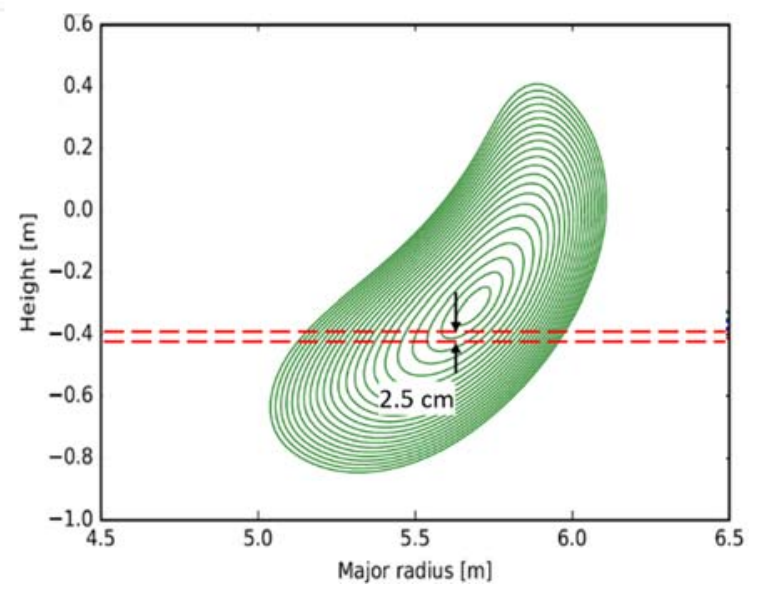

Fig. 3. The $1^{\text {st }}$ PHA channel line-of-sight (red dashes) with respect to flux surface data (green, from VMEC simulation) from magnetic configuration $\mathrm{J}$ (standard W7-X configuration during OP1.1) for the following geometry: distance plasma-pinhole $=7.4 \mathrm{~m}$, distance pinhole-detector $=0.97 \mathrm{~m}$, pinhole size $0.3 \times 0.3 \mathrm{~mm}$.

An energy calibration of the detector, based on fluorescence spectra, has been done [6]. The electronics associated with the detector consists of preamplifiers and a multichannel Digital Signal Processor (DSP) (manufactured by XIA [15]) that is common for all 3 PHA channels. By the combination of MCA (Multi-Channel Analyzer) bin width and MCA channels number during OP1.1 the energy range for the $1^{\text {st }}$ PHA channel was from 0.9 to $10 \mathrm{keV}$. The MCA bin width was set to $10 \mathrm{eV}$ while the MCA channels number was equal to 1024 .

The energy resolution of observed spectra was better than, or close to, $200 \mathrm{eV}$ depending on the DSP settings such as gap and peaking time. The latter setting is the time required for a shaped pulse to rise from the baseline to the peak while the gap time is the time between two pulses. The time resolution for PHA spectra in OP1.1 was $100 \mathrm{~ms}$ what was enough to collect spectra with quite good statistic and also to compare the results with other diagnostic with such a temporal resolution. Collected signals are line integrated along a line-of-sight that crosses close to the plasma center with spatial resolution of $2.5 \mathrm{~cm}$. Figure 3 presents the line of sight for the $1^{\text {st }}$ PHA channel. The plasma shape is calculated from the plasma equilibrium code VMEC using standard magnetic configuration J during OP1.1 [16].

\section{First PHA experimental results from $\mathrm{W} 7-\mathrm{X}$ plasmas}

Figure 4 presents examples of observed spectra for discharges with argon and neon puffs, as well as, for discharge without any gas-puff. For better identification of impurities lines, presented spectra are integrated over the whole discharges under similar experimental conditions from one experimental day. In fig.4c spectral lines are broader than lines presented in fig. $4 \mathrm{a}-\mathrm{b}$ due to $\mathrm{a}$ different peaking time value.

In the case of discharges with Ne-puff, the peaking time was short, $0.1 \mu$ s compared with its value for discharges without and with Ar-puff, $1 \mu \mathrm{s}$. All spectral lines from PHA spectra identified during the OP1.1 are listed in Tab. 1. It should be noted that only He-like and $\mathrm{H}$-like $\mathrm{S}, \mathrm{Cl}, \mathrm{Ne}$ and $\mathrm{Ar}$ lines were observed.

Table 1. List of identified impurity lines in W7-X plasmas during the OP1.1 campaign [17].

\begin{tabular}{lll}
\hline Type of line & $\mathrm{E}[\mathrm{keV}]$ & $\lambda[\AA]$ \\
\hline H-like Ne X $\left(\mathrm{Ne}^{+9}\right)$ & 1.022 & 12.13 \\
$\mathrm{H}-$-like Ne X $\left(\mathrm{Ne}^{+9}\right)$ & 1.211 & 10.24 \\
He-like S XV $\left(\mathrm{S}^{+14}\right)$ & 2.460 & 5.04 \\
H-like S XVI $\left(\mathrm{S}^{+15}\right)$ & 2.662 & 4.66 \\
He-like Cl XVI $\left(\mathrm{Cl}^{+15}\right)$ & 2.789 & 4.45 \\
H-like Cl XVII $\left(\mathrm{Cl}^{+16}\right)$ & 2.961 & 4.19 \\
He-like Ar XVII $\left(\mathrm{Ar}^{+16}\right)$ & 3.140 & 3.95 \\
He-like Cl XVI $\left(\mathrm{Cl}^{+15}\right)$ & 3.267 & 3.80 \\
H-like Ar XVIII $\left(\mathrm{Ar}^{+17}\right)$ & 3.321 & 3.73 \\
H-like Cl XVII $\left(\mathrm{Cl}^{+16}\right)$ & 3.508 & 3.53 \\
He-like Ar XVII $\left(\mathrm{Ar}^{+16}\right)$ & 3.684 & 3.37 \\
H-like Ar XVIII $\left(\mathrm{Ar}^{+17}\right)$ & 3.935 & 3.15 \\
\hline
\end{tabular}

An analysis of line intensities of particular elements, presented in Fig. 5, shows that intensities of sulphur (SXV@2.46keV) and chlorine (Cl XVI@2.789keV) do not vary significantly along a discharge while Ar line intensities (Ar XVII @3.140 keV and Ar XVIII @3.267 $\mathrm{keV}$ ), which was puffed into the plasma, change during a discharge with some decay time. This is clearly seen for 
the very intense Ar XVII line. In the case of Ar XVIII its intensity could be affected by the contribution of $\mathrm{Cl}$ XVI but still, a decay is observed. From their observed line intensity behavior, it seems that $\mathrm{Cl}$ and $\mathrm{S}$ are the intrinsic impurities.
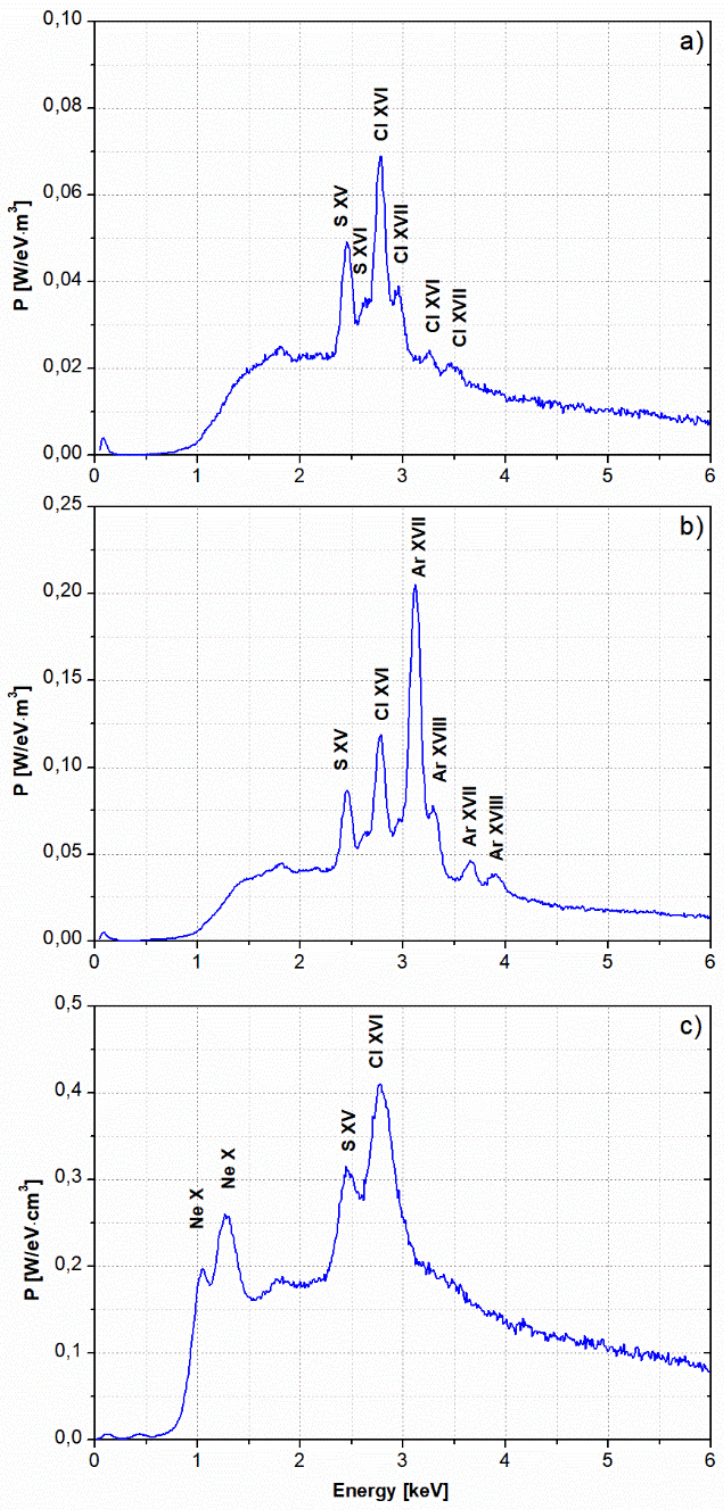

Fig.4. An example of observed spectra for the W7-X discharges with no gas-puff (a) (discharges from 2016.03.09), with an Ar puff (b) (discharges from 2016.03.09) and with a Ne puff (c) (discharges from 2016.03.08). Identified spectral lines are highlighted. (spectra are not corrected for the detector efficiency).

This is consistent with the assumption of a constant source for the $\mathrm{S}$ and $\mathrm{Cl}$ and a short source pulse followed by a decay which is determined by a convolution of the impurity confinement time and recycling of Ar.

It is worth to add that material analysis of limiter tails, before and after their exposure to W7-X plasma, was done [18]. An Energy-Dispersive X-ray (EDX) scan shows that, as well as oxygen and nitrogen (the 1st PHA channel is not sensitive in the energy range of these elements), both chlorine and sulphur were deposited on the limiter surface [18]. In the past, $\mathrm{Cl}$ and $\mathrm{S}$ were observed in other fusion devices like JET [19] or Alcator-C [20-21]. The source of these elements in W7-X is still unclear but rough estimations shows that their concentration is quite small and they do not contribute significantly to total radiation losses. A speculative reason for the presence of these elements could be a left behind PVC element e.g. a protection cap on a bolt. In the next experimental campaign, which will start after the installation of the inertially cooled divertor (Test Divertor Unit, TDU) and after checking the whole machine, it is expected that these impurities will no longer be observed.
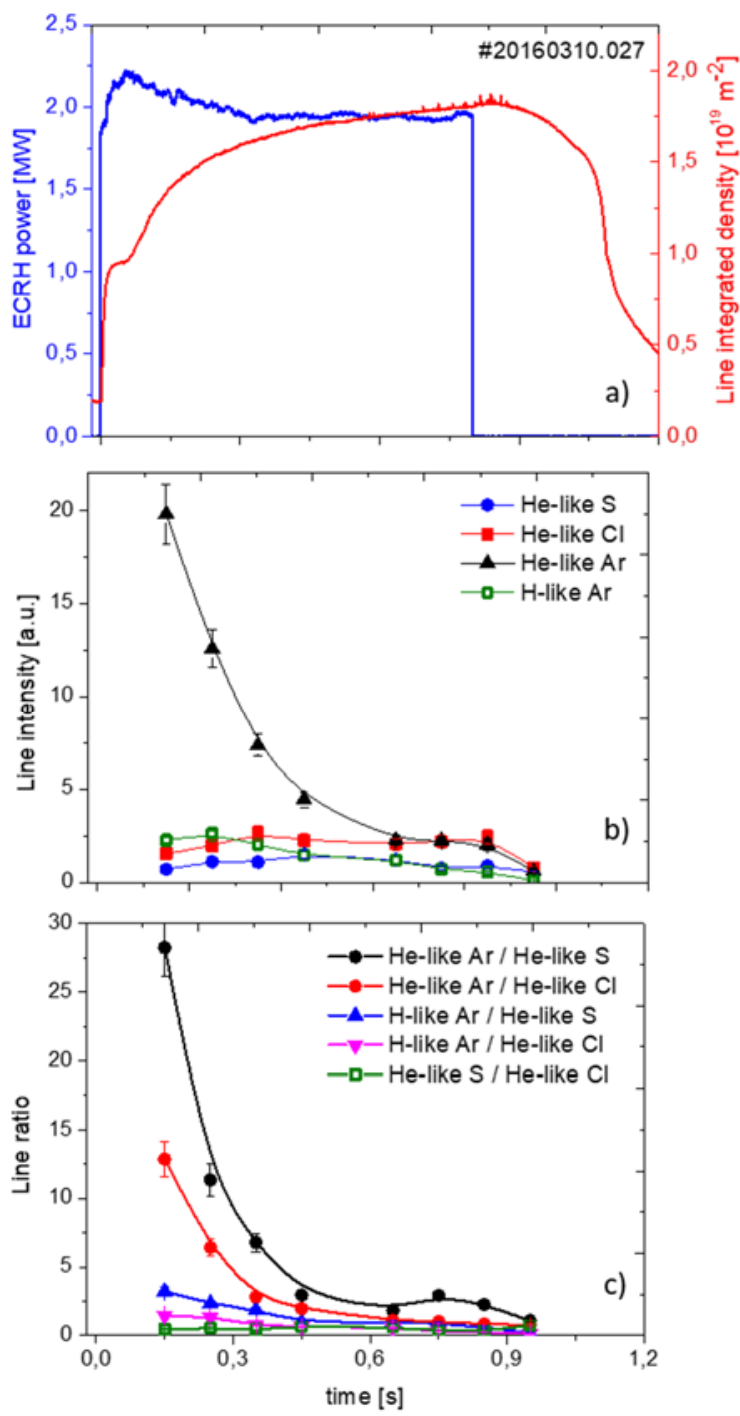

Fig.5. Time evolution of a) ECR heating power and line integrated electron density, b) impurity line intensities, c) line-ratios during the particular W7-X discharge \# 20160310.027 .

Detailed studies of impurity behavior in W7-X is important in order to investigate e.g. the impact of different magnetic field configurations on impurity core and edge transport [22]. For this reason, the PHA system, with its good energy resolution, is significant W7-X diagnostic. Indeed, the impurities observed by the PHA system has been also confirmed by other diagnostics, e.g. by the High-Efficiency XUV Overview Spectrometer HEXOS [23-24] which is sensitive to the line emission of medium and low ionization states of these species. Figure 6 presents an example of a spectrum observed by HEXOS. 
In relation to the $\mathrm{C}$ and $\mathrm{O}$ lines also identified in the spectrum (the content on $\mathrm{C}$ and $\mathrm{O}$ is higher than content of $\mathrm{S}$ and $\mathrm{Cl}$ in the plasma), the intensity of $\mathrm{Cl}$ and $\mathrm{S}$ lines is very small.

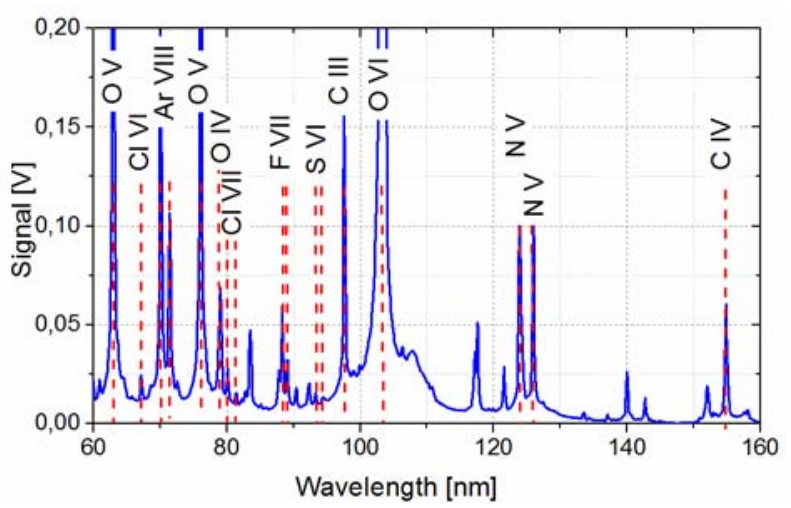

Fig.6. An example of a spectrum with identified lines observed by HEXOS diagnostics (spectrometer 4). Not assigned lines are not yet identified.

In the case of PHA spectra collected by the $1^{\text {st }}$ channel, $\mathrm{C}$ and $\mathrm{O}$ are not observed (their positions are below the $1^{\text {st }}$ PHA channel lower energy range, e.g. O VIII $653.4 \mathrm{eV}$, O VII 573.9 eV, C VI 367.5 eV, C V 307.9 eV [17]), therefore the spectrum is dominated by the $\mathrm{Ar}, \mathrm{Cl}$ and $\mathrm{S}$ lines. In the next experimental campaign, OP1.2a, when the $3^{\text {rd }}$ PHA channel will be optimized and collect spectra with light impurities lines, it will be possible to compare signal intensities of observed lines with other diagnostics.

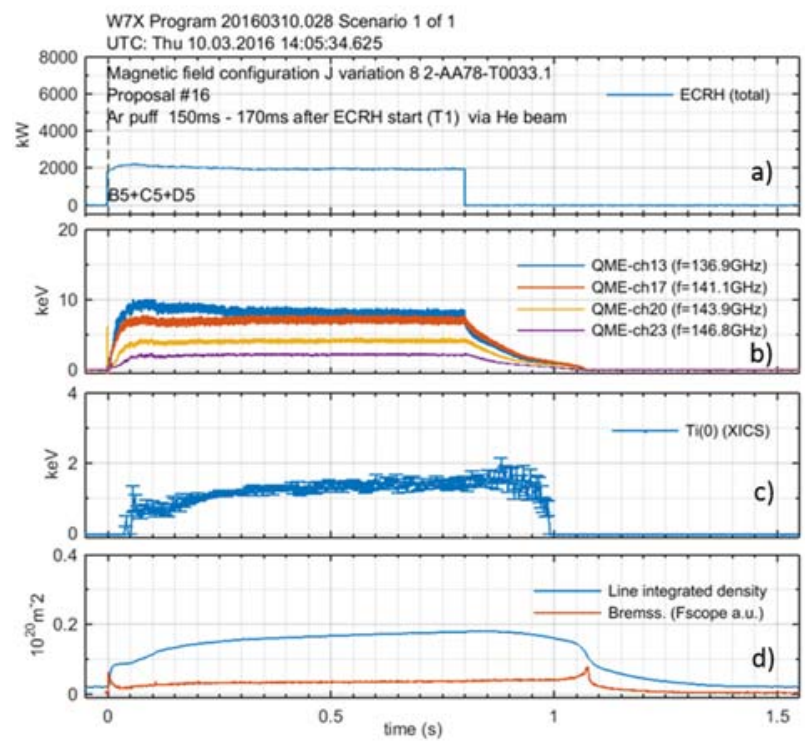

Fig.7. The waveform of the plasma discharge 20160310.028: a) ECRH power, b) the electron temperature (ECE), c) the ion temperature (XICS), d) line integrated electron density.

It is envisaged that the PHA system will be a routine diagnostic for $\mathrm{T}_{\mathrm{e}}$ estimation on W7-X. Thus, the spectra collected during the OP1.1 campaign were analyzed to deliver average, line-of-sight, electron temperatures. The continuous part of the PHA spectra consists of bremsstrahlung and recombination radiation, both of which are $T_{e}$ dependent $[10,25-26]$. From the slope of intensity versus energy of the continuum average over the
PHA system line of sight, $\mathrm{T}_{\mathrm{e}}$ is estimated. An example of collected PHA spectra at different time intervals along a discharge with Ar puff (see waveform in Fig.7) is presented in Fig.8. In this case, the $T_{e}$ is determined from the slope of the continuum for energies greater than $4 \mathrm{keV}$ (to select region without spectral lines).

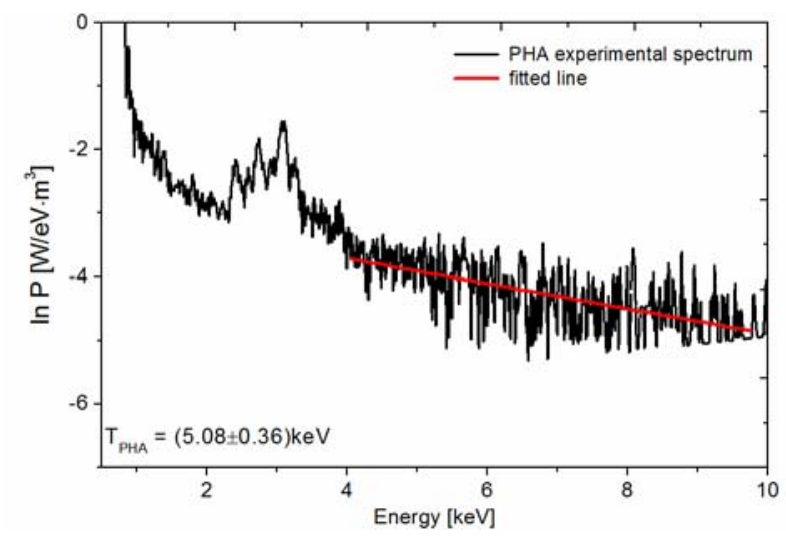

Fig.8. An example of the PHA spectrum in the logarithmic scale for the discharge with Ar puff (\#20160310.028). The spectrum corresponds to 550ms after the ECRH.

Changes of electron temperature calculated from the PHA spectra during the discharge with $2 \mathrm{MW}$ of ECRH are presented in Fig.9. A comparison of estimated $\mathrm{T}_{\mathrm{e}}$ values could be done e.g. by using a correction factor evaluated from the modeling of the continuum radiation for central electron temperature [9]. A second option is to simulate spectra using experimental $\mathrm{T}_{\mathrm{e}}$ and $\mathrm{n}_{\mathrm{e}}$ profiles e.g. from the Thomson Scattering diagnostic, and then estimate $T_{e}$ from the slope of continuum radiation. Such an approach of PHA spectra analysis is presented in this reference [27].

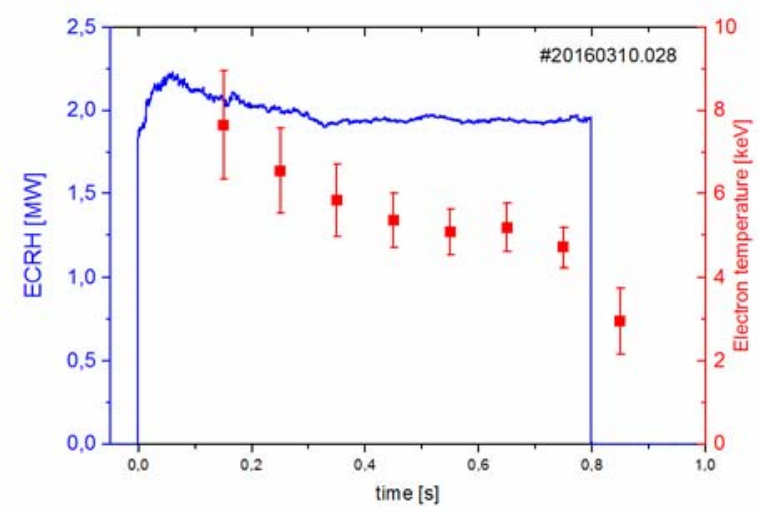

Fig.9. Calculated from the PHA spectra $T_{e}$ values for different time instants of W7-X discharge \#20160310.028.

The PHA spectra can also deliver information about the average effective charge, $Z_{\text {eff. }}$ The procedure is based on comparison of experimental and theoretical spectra [910]. For this purpose, a special numerical code based on RayX code [28], is being developed to estimate $Z_{\text {eff }}$ values of W7-X plasmas.

\section{Summary}

During the first experimental campaign at W7-X stellarator, a PHA system has been commissioned and tested. The data obtained from its $1^{\text {st }}$ PHA channel have 
been analyzed to deliver information about impurity plasma content and to estimate a line-averaged $\mathrm{T}_{\mathrm{e}}$. He-like and $\mathrm{H}$-like lines of $\mathrm{S}, \mathrm{Cl}$, Ar and $\mathrm{Ne}$ have been identified. Spectral lines corresponding to heavier impurities have not been observed. The obtained count rate levels together with energy resolution make the W7-X PHA system a useful diagnostic tool for impurity transport studies and electron temperature estimation. In the next step, the analysis of collected signals will focus on comparisons of experimental PHA spectra with simulated ones to estimate impurity concentrations.

For the next phase of W7-X operation it is planned to optimize all three PHA channels to observe lines from light elements, e.g. carbon, nitrogen and oxygen. This will be possible in the case of the $3^{\text {rd }}$ PHA channel which is equipped in SDD detector covered with a thin Polymer window (AP3 window from Moxtek company [29]). Additionally, the use of thicker Be filters in the $1^{\text {st }}$ PHA channel (which reduce photons originating from the low energy region) together with increasing the slits widths, could give information about higher $Z$ elements in the plasma.

\section{Acknowledgments}

This work has been carried out within the framework of the EUROfusion Consortium and has received funding from the Euratom research and training programme 20142018 under grant agreement No 633053. The views and opinions expressed herein do not necessarily reflect those of the European Commission.

This scientific work was partly supported by Polish Ministry of Science and Higher Education within the framework of the scientific financial resources in the years 2014-2017 allocated for the realization of the international co-financed project.

\section{References}

[1] H.-S. Bosch et al., Technical challenges in the construction of the steady-state stellarator Wendelstein 7-X Nuclear Fusion 53 (2013) 126001

[2] T. Sunn Pedersen et al., Plans for the first plasma operation of Wendelstein 7-X, Nuclear Fusion 55 (2015) 126001

[3] H.-S.Bosch et al. Physics Programme for Initial Operation of Wendelstein 7-X, Contribution to Plasma Physics 50, No. 8 (2010) 687

[4] R. Koenig et al., The Set of Diagnostics for the First Operation Campaign of the Wendelstein 7-X Stellarator, Journal of Instrumentation 10 (2015) P10002

[5] M. Krychowiak et al., Overview of diagnostic performance and results for the first operation phase in Wendelstein 7-X, Review of Scientific Instruments 87 (2016) 11D304

[6] M. Kubkowska et al., Laboratory tests of the Pulse Height Analysis system for Wendelstein 7-X, Journal of Instrumentation 10 (2015) P10016

[7] N. Krawczyk et al., Commissioning and first operation of the pulse-height analysis diagnostic on Wendelstein 7-X stellarator, Fusion Engineering and Design 123 (2017) 1006-1010

[8] Z.Y. Chen et al., A compact soft X-ray PHA in the HT-7 tokamak, Nuclear Instruments and Methods in Physics Research A 527 (2004) 604-608

[9] A. Weller et al., X-ray Pule Height Analysis on Asdex Upgrade, $38^{\text {th }}$ EPS Conference on Plasma Physics (2011) P5.054

[10] D. Pasini et al., JET X-ray pulse-height analysis system, Review of Scientific Instruments 59 (1988) 693-699

[11] S. Muto et al., First results from X-ray pulse height analyser with radial scanning system from LHD, Review of Scientific Instruments 27 (2001) 1206-

[12] T. Klinger et al., Performance and properties of the first plasmas of Wendelstein 7-X, Plasma Physics and Controlled Fusion 59 (2017) 014018 (8pp)

[13] R.C. Wolf et al., Major results from the first plasma campaign of the Wendelstein 7-X stellarator, Nuclear Fusion 57 (2017) 102020 (13pp)

[14] http://pndetector.de/

[15] http://www.xia.com

[16] T. Sunn Pedersen et al., Confirmation of the topology of the Wendelstein 7-X magnetic field to better than 1:100,000, Nature communications 7 (2016) 13493

[17] http://physics.nist.gov/PhysRefData/ASD/ionEnergy.html

[18] V. Winters et al., Overview of the plasma-surface interaction analysis in the limiter phase of W7-X, Physica Scripta T170 (2017) 014050

[19] JET Joint Undertaking Progress Report 1984 http://aei.pitt.edu/53413/1/JET.J.U.1984.pdf

[20] E. Kallne et. al, Observation of H- and He-like x-ray line emission in high density tokamak plasmas, Physical Review Letters 49 (1982) 330-333

[21] J. E. Rice et al., The Rydberg series of helium-like Cl, Ar and $\mathrm{S}$ and their high-n satellites in tokamak plasmas, New Journal of Physics 1 (1999) 19.1-19.27

[22] A. Czarnecka et al., Study of impurities behaviour in PHA spectra for first magnetic configuration changes in W7-X plasmas, $19^{\text {th }}$ ISFNT, Kyoto, Japan 2017

[23] H. Thomsen et al., Startup impurity diagnostics in Wendelstein 7-X stellarator in the first operational phase, Journal of Instrumentation 10 (2015) P10015

[24] B. Buttenschön et al., Spectroscopic impurity survey in the first operation phase of Wendelstein 7-X, Proceedings of $43^{\text {rd }}$ EPS Conference on Plasma Physics, P4.012, Vol. 40A, ISBN:2-914771-99-1

[25] S. von Goeler et al., Thermal X-ray spectra and impurities in the ST Tokamak, Nuclear Fusion 15 (1975) 301-311

[26] R. D. Gill et al., Soft X-ray measurements of the impurity density in DITE, Nuclear Fusion 19 (1979) 1003-1010

[27] N. Krawczyk et al., Electron temperature estimation using the Pulse Height Analysis System at Wendelstein 7-X Stellarator, 19th ISFNT, Kyoto, Japan 2017

[28] S. Jabłoński et al., Simulation of pulse height analysis soft X-ray spectra expected from W7-X, Journal of Instrumentation 10 (2015) P10021

[29] www.moxtek.com 